\title{
An adaptive motion planning algorithm for obstacle avoidance in autonomous vehicle parking
}

\author{
Naitik M. Nakrani ${ }^{1}$, Maulin M. Joshi ${ }^{2}$ \\ ${ }^{1}$ Department of Electronics and Communication Engineering, Uka Tarsadia University, India \\ ${ }^{2}$ Department of Electronics and Communication Engineering, Gujarat Technological University, India
}

\begin{abstract}
Article Info
Article history:

Received Dec 3, 2020

Revised Apr 14, 2021

Accepted May 15, 2021

\section{Keywords:}

Adaptive fuzzy systems

Autonomous vehicles

Dynamic path planning

Potential field

Ultrasonic sensing

ABSTRACT

In the recent era, machine learning-based autonomous vehicle parking and obstacle avoidance navigation have drawn increased attention. An intelligent design is needed to solve the autonomous vehicles related problems. Presently, autonomous parking systems follow path planning techniques that generally do not possess a quality and a skill of natural adapting behavior of a human. Most of these designs are built on pre-defined and fixed criteria. It needs to be adaptive with respect to the vehicle dynamics. A novel adaptive motion planning algorithm is proposed in this paper that incorporates obstacle avoidance capability into a standalone parking controller that is kept adaptive to vehicle dimensions to provide human-like intelligence for parking problems. This model utilizes fuzzy membership thresholds concerning vehicle dimensions and vehicle localization to enhance the vehicle's trajectory during parking when taking into consideration obstacles. It is generalized for all segments of cars, and simulation results prove the proposed algorithm's effectiveness.
\end{abstract}

This is an open access article under the CC BY-SA license.

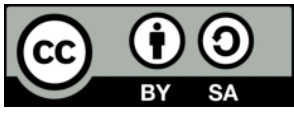

\section{Corresponding Author:}

Naitik M. Nakrani

Department of Electronics and Communication Engineering

Uka Tarsadia University

Bardoli-Mahuva Road, Surat, India

Email: naitiknakrani@gmail.com

\section{INTRODUCTION}

Autonomous parking systems and navigation issues have attracted much research recently due to the development of autonomous vehicle technologies. One prominent reason for using this technique is to improve human performances via advanced robotic technologies, such as 'smart parking' and 'smart highways'. In modern times, these technologies demand that autonomous robots function intelligently as a human in an unknown and unstructured environment. A mobile robot must sense its surroundings and determine appropriate actions to work in such under-constrained, dangerous, and dynamic domains.

Autonomous parking involves proper path planning and control for a car-like mobile robot (CLMR) without human intervention. Parking is considered a challenging task for a machine due when the environment considered is dynamic and uncertain. In such an environment, online parking systems give better performance compared to traditional offline parking systems. Online parking systems carried out path planning in a parallel while; i) moving towards a goal and ii) perceiving the environment. This perceived information is used in multiple ways in parking systems, e.g., environment mapping, parking space detection, obstacle detection and avoidance, collision detection, and following to walls. Past studies involved different types of sensors in the parking system for various purposes. In earlier work, [1] presented a skill-based fuzzy logic approach for forward and reverse parking. They used six infrared sensors to achieve wall following behavior and past parking posture correction. Another skill-based fuzzy logic approach is given in [2]. Their algorithm generates 
the parking path based on the vehicle's position and orientation relative to the parking space. It is designed for a tight parking space, and sensors are utilized to detect parking dimensions and ready to reverse points for proper parking. Demirli and Khoshnejad [3] used a fifth-order polynomial path data along with sensors for training their adaptive neuro-fuzzy control algorithm. This approach does not require a reference path, and sensors provide nearby information to adapt parameters in the network. However, the system is built on the assumption of having a large parking space and a mostly obstacle-free environment. A fuzzy logic-based parking system is also used in [4]. They utilized infrared sensors for detecting parking spaces. A multifunctional parking controller is presented in [5] using a skill-based fuzzy logic control. They used infrared sensors to detect obstacles in the forward path. This system is designed to handle environment dynamicity, but its outcome is limited. As their system does not have any target steer functionality, it bypasses the obstacle and the parking space even though the path is available for parking. An ultrasonic sensor-based fuzzy control system is used in [6]. Two model predictive control schemes for autonomous parallel parking are developed in [7], [8]. Their path tracking method claims improved accuracy and stability compared to fuzzy logic but increases the computational burden. Similar to previous methods, both of these approaches utilize their sensors to detect a parking slot or avoid curbs and corners of the parked vehicles along with the parking space. A similar approach can also be found in [9]. All of these methods utilize a fuzzy logic theory to implement human intelligence linguistically. These systems are built on the assumption that the environment is fully or partially known and slowly moving. Also, the systems do not consider the impact on the system's performance compared to the vehicle's non-holonomic dimensions. We aim to address both the challenges in this work by utilizing the knowledge of the navigation problems and integrating it into the existing parking architectures.

The solution of an autonomous parking system in a dynamic environment can be achieved by doing motion planning with the navigation algorithm. Many behavior-based reactive navigation architectures can be found [10]-[17] in literature. In earlier work, [18] proposed dynamic path planning for non-holonomic CLMRs using the bubble band concept. A human heuristics-based rule base for reactive navigation is generated in [19]. Joshi and Zaveri [20] proposed a velocity control-based fuzzy navigation controller using ultrasonic sensors for the dynamic environment. The artificial potential field has been widely used for collision-free obstacle avoidance in a known environment. Non-holonomic vehicle navigation through narrow passage was developed in [21] using a potential field. A fuzzy-based potential field method was presented in [22] for dynamic motion planning, including static and moving obstacles. A modified bacterial potential field was proposed in [23] for dynamic motion planning. A fuzzy logic theory optimized by the PSO algorithm is integrated with the potential field for dynamic motion planning in [24]. A sampling-based motion planning rapidly-exploring random tree (RRT) and the potential field was presented in [25] for autonomous valet parking. In another study, an optimization [26] and artificial potential field [27] based mobile robot navigation are presented.

A professional human driver can maneuver between ideal gaps with little knowledge of obstacles and an understanding of vehicle dynamics. When driving a compact hatchback or a big special utility vehicle, the drive-through experience is different. Correlation of such human driving skills with different segments of vehicles required adaptive changes in the design specification of the fuzzy inference system. So, for obstacle avoidance, one set of fuzzy membership functions used in literature may not work for all different dimensions of the vehicle. One must note that the perceptual inference of distances in a similar environment by various vehicles with distinct dimensions is different, as conceptually shown in Figure 1(a)-(c). It reveals an important fact that a fixed and predetermined set of thresholds used in the existing fuzzy-based vehicle control system must be made adaptive by considering vehicle size parameters.

In this paper, we propose a unique approach for adaptation of the fuzzy inferences using the potential field approach for obstacle avoidance. Our proposed adaptive motion planning controller utilizes a parameter derived from the vehicle's actual dimensions and then provides the capability to adapt fuzzy variable membership values and successfully park different size cars. In earlier work [28], a hybrid intelligent autonomous vehicle parking system with an inbuilt obstacle avoidance module was established. We incorporate our proposed algorithm into the parking module in this paper. Simulations results are generated to understand the impact of the vehicle dimensions on the motion planning and a possible way to make it generalized for autonomous parking infrastructures like valet parking, and on-street parallel parking. The use of an adaptive fuzzy system removes the local minima's limitation in the potential field due to the adaptation.

This paper's organization is: in section 2, a hybrid autonomous parking system architecture is explained, and it is followed by an explanation of the proposed adaptive motion planning algorithm. In section 3, simulation results are given for the different dimensions of the vehicle and show the proposed algorithm's efficacy. Finally, the conclusions are given in section 4. 


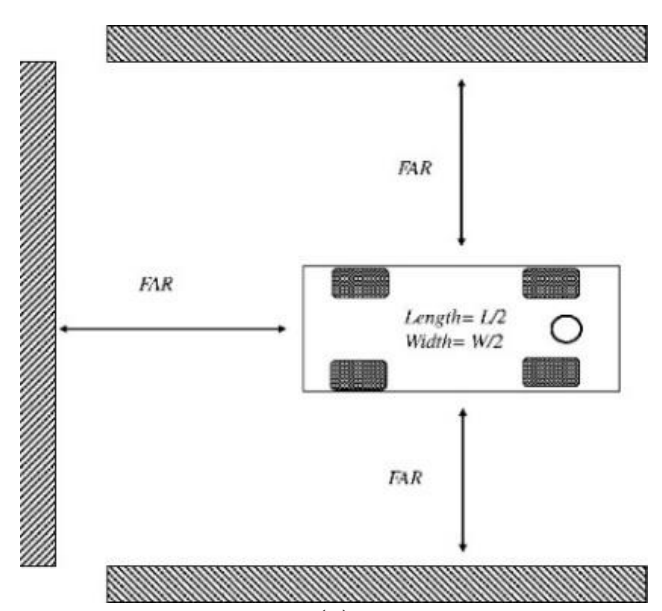

(a)

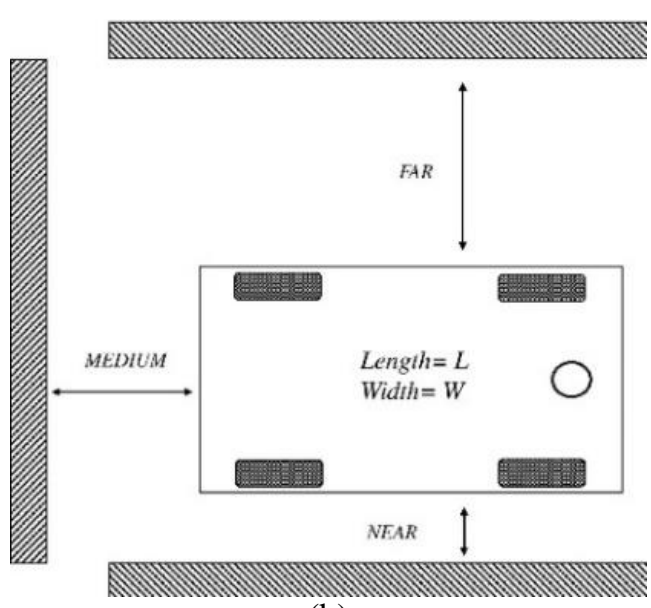

(b)

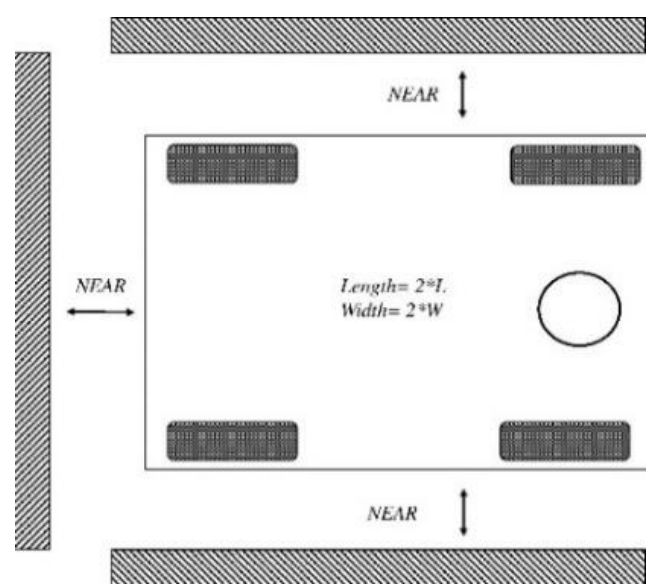

(c)

Figure 1. A typical 'U shape' scenario indicating dynamic (different) inference of the same environment by different sized vehicles: (a) small, (b) medium, and (c) large

\section{OVERALL HYBRID AUTONOMOUS PARKING SYSTEM ARCHITECTURE}

An Intelligent autonomous parking system integrating the proposed algorithm is developed, as shown in Figure 2. It consists of three major components, a parking controller, an adaptive motion controller, and a decision controller. The primary task of this system is to park a vehicle in a parallel mode in a dynamic environment. A dynamic environment may contain several obstacles. The vehicle must avoid them intelligently and perform parking. Figure 2 shows an adaptive motion planning controller integrated with a parking controller to accomplish this task. A parking controller is dedicated to parallel parking, and an adaptive motion planning controller is designed for obstacle avoidance. The controlling outputs of both controllers are passed to the vehicle depending upon the requirement of the task. A decision module controls the output of both controllers. Based on the decision module conditions, it will pass either of these two outputs $\left(\varphi_{1}\right.$ and $\left.\varphi_{2}\right)$ shown in the block diagram. The information gathered by the ultrasonic sensors mounted on the vehicle's perimeter plays a vital role in the adaptive motion planning controller and the decision module.

The architecture shown in Figure 2 is a decentralized multi-level fuzzy system. It is designed for multi-tasking such as parallel parking, environment sensing, obstacle avoidance, wall following, target steer, and parking space detection. With a large number of inputs, the rule-based for a different fuzzy system can be designed separately and optimally, which makes our system relatively faster, reliable, and computationally efficient compared to other fuzzy and nonlinear control mechanisms found in the literature for parking. Also, integration of motion planning controller with parking controller can perform obstacle avoidance with parking in an unknown and dynamic environment. It remains a major challenge in this domain.

Our proposed adaptive motion planning controller is a four-input one output fuzzy logic controller. Its rule-base is designed for the task of obstacle avoidance. It is developed from the classical knowledge of navigation for the mobile robot. In [28], the basic rule-base for the obstacle avoidance and parallel parking controller is proposed. However, their design was fixed and invariant like major of the navigation literature. In 
this paper, its design is made adaptive with the shape of the non-holonomic vehicle. The system can be adaptively used for any size of the non-holonomic vehicle. The controllers' membership functions are made variable with respect to the length and the width of the vehicle. Also, it is made adaptive during runtime concerning the sensor information for optimized performance. In the following subsection, the proposed algorithm is explained in detail.

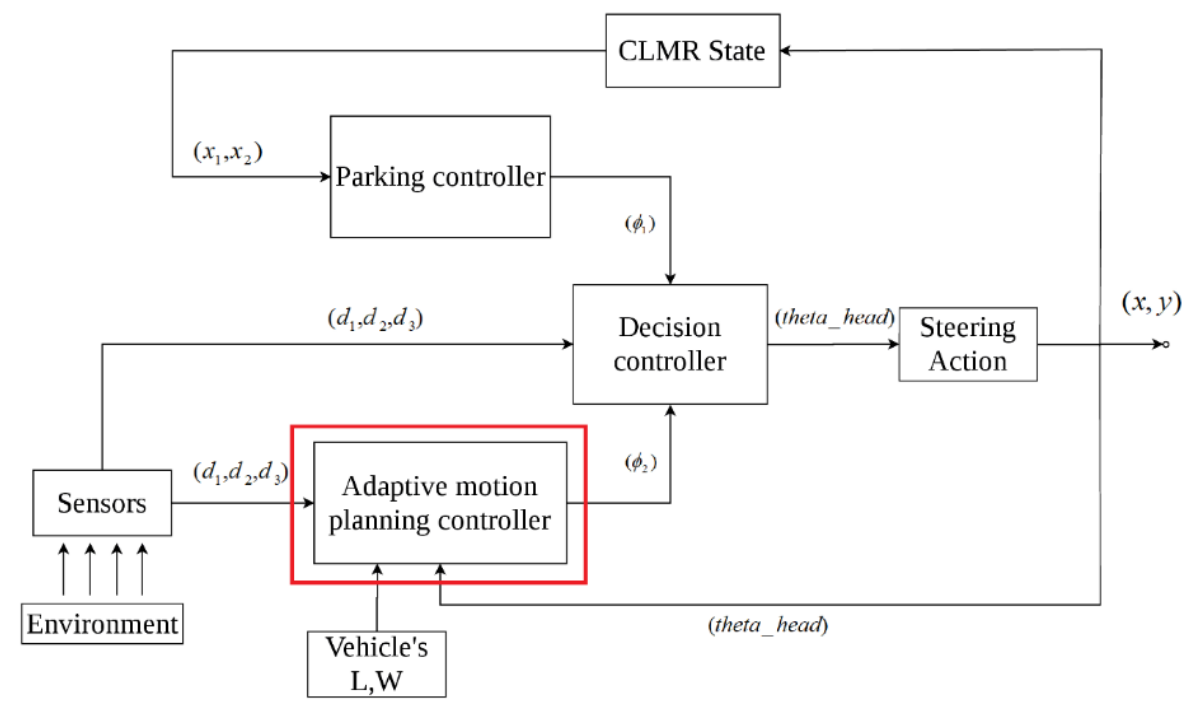

Figure 2. A hybrid autonomous parking system architecture

For environment sensing, a total of 12 ultrasonic sensors are assumed to be mounted on the perimeter of the vehicle, as shown in Figure 3. To utilize the collective information of a large number of ultrasonic sensors, they are grouped in three categories: left side sensor group $\left(\mathrm{d}_{1}\right)$, front/rear side sensor group $\left(\mathrm{d}_{2}\right)$ and, right side sensor group $\left(d_{3}\right)$. For the forward parking, the front side of the sensors Figure 3(a) is used, and for the backward parking, the rear side of the sensors Figure 3(b) is used. Each sensor gives distance value from the nearby obstacles, and to use them effectively; they are grouped as per (1).

$$
\begin{aligned}
& d_{1}=\min \left\{d i s t \_S_{1}, d i s t \_S_{2}, d i s t_{-} S_{3}\right\} \\
& d_{2}=\min \left\{d i s t \_S_{3}, \text { dist_}_{-} S_{4}, \text { dist_ } S_{5}\right\} \\
& d_{3}=\min \left\{d i s t \_S_{5}, \text { dist_}_{-} S_{6}, \text { dist_}_{-} S_{7}\right\}
\end{aligned}
$$

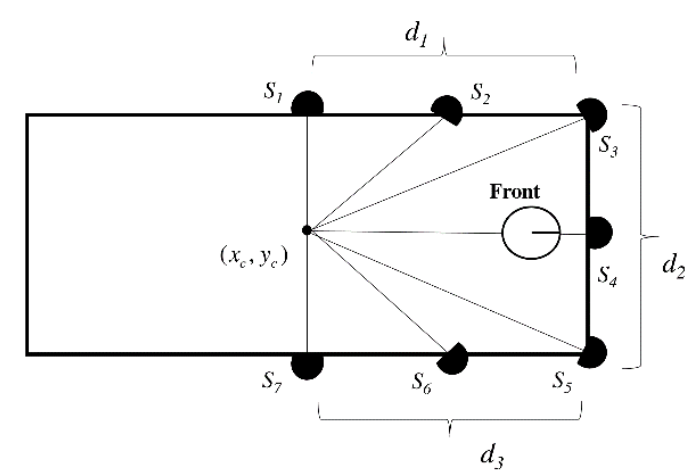

(a)

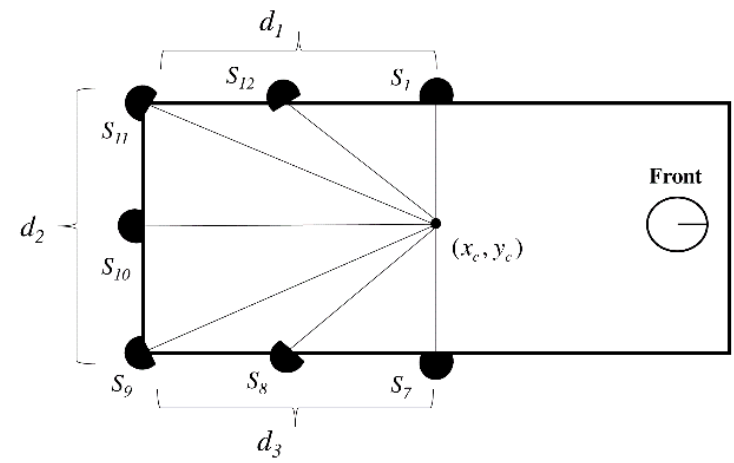

(b)

Figure 3. Ultrasonic sensor placement and grouping on the vehicle; (a) front side and (b) rear side

A similar set of equations can be assumed for backward parking with different sensor groups, as shown in Figure 3(b). This approach establishes better sensing using a large number of sensors and effective 
reduction of the inputs for the system with groups. In our earlier work, these grouped inputs were fuzzified into three different membership functions with fix set of their threshold values and ranges. With our proposed algorithm, these thresholds are made variable and adaptive with the non-holonomic vehicle size. The behavior logic of the decision module can be summarized as pseudo-code explained by:

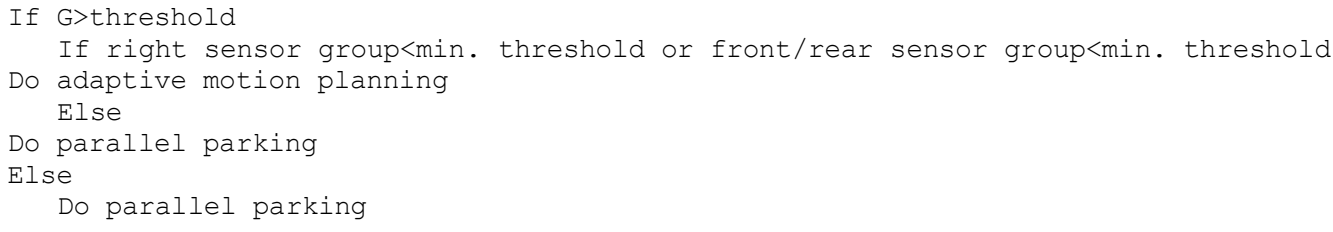

Here, $\mathrm{G}$ defines the Euclidean distance between the target point and the current location of the vehicle. When obstacles are detected in the environment during a parking maneuver, the decision controller will switch the vehicle's command to the adaptive motion planning controller. The adaptive motion planning controller will detour the vehicle safely away from the obstacles and avoid any collision considering the safe drivable area around the vehicle.

\subsection{Proposed motion planning algorithm}

- $\quad$ Let CLMR be with the size of length L and width W.

- $\quad$ Let the minimum safety distances are defined as per the geometry of the CLMR's size, as shown in Figure 4.

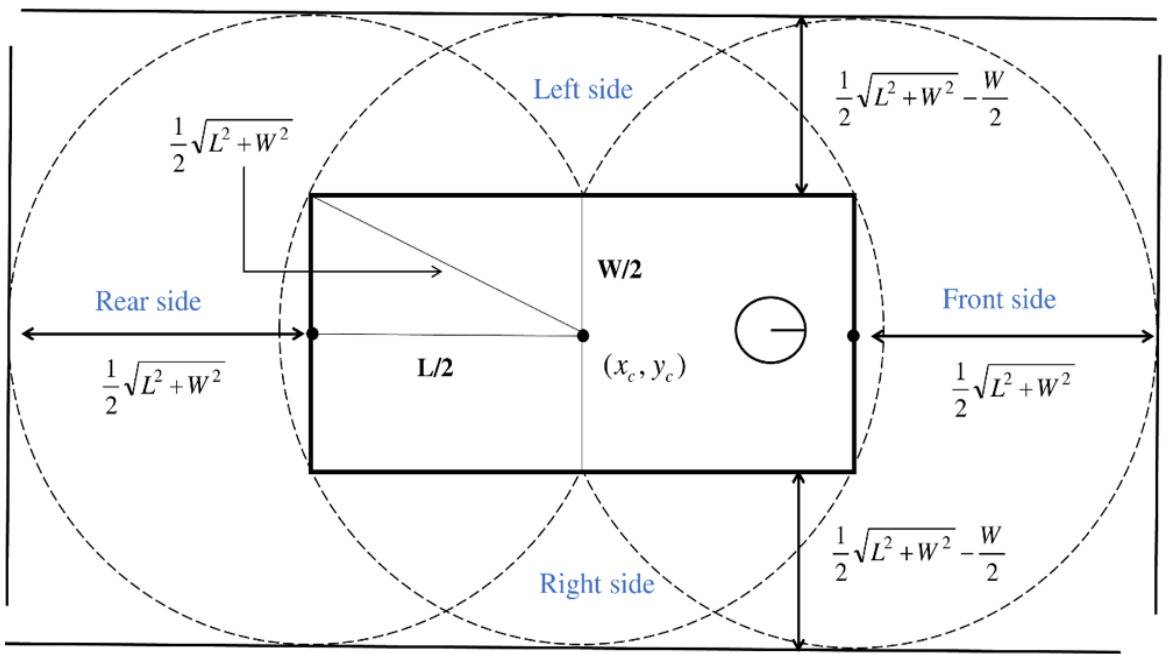

Figure 4. CLMR geometry and Safe away distance boundary from the obstacle

- $\quad$ Let the three distance groups defined in (1) are fuzzified into three different membership functions Near, Medium and, Far.

- $\quad$ Let the thresholds of $\mathrm{d}_{1}$ and $\mathrm{d}_{3}$ are defined as:

$$
\begin{aligned}
& \mu_{\text {near }}(x)=\operatorname{trap}\left(-R_{1}, 0, R_{1}-\frac{R_{1}}{4}, R_{1}\right) \\
& \mu_{\text {med }}(x)=\operatorname{trig}\left(R_{1},-\frac{R_{1}}{4}, R_{1}, R_{1}+\frac{R_{1}}{4}\right) \\
& \mu_{\text {far }}(x)=\operatorname{trap}\left(R_{1}, R_{1}+\frac{R_{1}}{4}, 3 R_{1}, 4 R_{1}\right)
\end{aligned}
$$

where, $R_{1}=\frac{1}{2} \sqrt{L^{2}+W^{2}}-\frac{W}{2}$ and $x \in\left\lfloor 0,3 R_{1}\right\rfloor$ for $\mathrm{d}_{1}$ and $\mathrm{d}_{3}$

- Similarly, let the thresholds of $\mathrm{d}_{2}$ is defined as:

$$
\mu_{\text {near }}(x)=\operatorname{trap}\left(-R_{2}, 0, R_{2}-\frac{R_{2}}{4}, R_{2}\right)
$$




$$
\begin{aligned}
& \mu_{\text {med }}(x)=\operatorname{trig}\left(R_{2},-\frac{R_{2}}{4}, R_{2}, R_{2}+\frac{R_{2}}{4}\right) \\
& \mu_{\text {far }}(x)=\operatorname{trap}\left(R_{2}, R_{2}+\frac{R_{2}}{4}, 3 R_{2}, 4 R_{2}\right)
\end{aligned}
$$

where, $R_{2}=\frac{1}{2} \sqrt{L^{2}+W^{2}}-\frac{W}{2}$ and $x \in\left\lfloor 0,3 R_{2}\right\rfloor$ for $\mathrm{d}_{2}$.

- $\quad$ These thresholds are varied based on the length and width of the vehicle. These values are smaller for the small hatchback-sized car, and for the large sedan and luxurious sized car, these values are larger.

\subsection{Adaptive dimension algorithm}

- We define three constants $\mathrm{K}_{1}, \mathrm{~K}_{2}$, and $\mathrm{K}_{3}$ for the three distances $\mathrm{d}_{1}, \mathrm{~d}_{2}$ and, $\mathrm{d}_{3}$. These constants are used to modify thresholds of fuzzy membership functions adaptively. Let their range be like (8).

$$
\frac{W}{2 L} \leq K_{i} \leq \frac{L}{W}
$$

- $\quad$ To modify thresholds, multiply this factor ' $K_{i}$ ' with a threshold range of fuzzy membership functions,

$$
\mu_{\text {near }}(x)=\operatorname{trap}\left(a^{\prime}, b^{\prime}, c^{\prime}, d^{\prime}\right)
$$

where

$$
\begin{aligned}
& a^{\prime}=K_{i} \times a ; b^{\prime}=K_{i} \times b ; c^{\prime}=K_{i} \times c ; d^{\prime}=K_{i} \times d \\
& \mu_{\text {med }}(x)=\operatorname{trig}\left(a^{\prime}, b^{\prime}, c^{\prime}\right)=\operatorname{trig}\left(K_{i} \times a, K_{i} \times b, K_{i} \times c\right) \\
& \mu_{\text {far }}(x)=\operatorname{trap}\left(a^{\prime}, b^{\prime}, c^{\prime}, d^{\prime}\right)=\operatorname{trap}\left(K_{i} \times a, K_{i} \times b, K_{i} \times c, K_{i} \times d\right)
\end{aligned}
$$

where, $i=1,2,3$ for the distances $d_{1}, d 2, d 3$

- We define a change in factor ' $\mathrm{K}_{\mathrm{i}}$ ' from the knowledge of repulsive potential field used in motion planning problems [18], [25],

$$
\Delta K_{i}=\left\{\begin{array}{l}
-\frac{\eta}{2}\left(\frac{1}{d_{i}}-\frac{1}{d_{i s}}\right)^{2} \text { if } d_{i}>d_{i s} \\
\frac{\eta}{2}\left(\frac{1}{d_{i}}-\frac{1}{d_{i s}}\right)^{2} \text { if } d_{i}>d_{i s}
\end{array}\right.
$$

Here, $\eta$ is the learning rate, and $d_{i s}$ is min. safe distance for each distance group $d_{1}, d_{2}$ and $d_{3}$.

- $\quad$ Algorithm 1 is used to modify thresholds of the distances used for obstacle avoidance with adaptive ' $\mathrm{K}_{\mathrm{i}}$ '. As the vehicle approaches near to the obstacle, $d_{i}$ is reduced, and $\Delta K_{i}$ reduces $K_{i}$. If any distance goes below $\mathrm{d}_{\mathrm{is}}, \Delta \mathrm{K}_{\mathrm{i}}$ becomes positive, and that increases $\mathrm{K}_{\mathrm{i}}$. Hence it maintains the minimum safe distances from the obstacle.

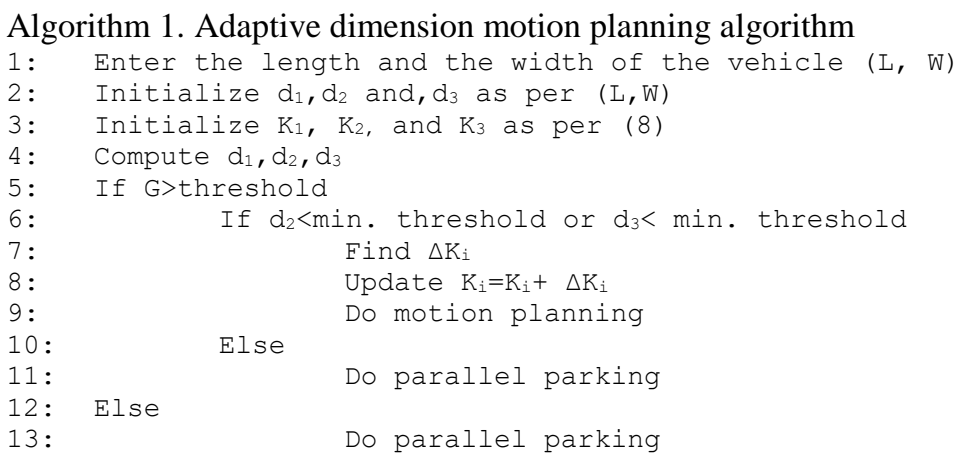

- $\quad$ This algorithm adapts membership functions of the input distances as per the size of the vehicle. The smaller vehicle has the smaller value of ' $K$ ', and the larger vehicle has, the larger value of ' $\mathrm{K}$ '. This adaptive dimension motion planning controller makes our design very intelligent and novel. 
- The value of $\mathrm{d}_{\text {is }}$ is the minimum safe distance one has to achieve for autonomous driving. It can be select heuristically or experimentally depending upon the requirement.

\section{SIMULATION RESULTS}

This work aims to show the impact of the vehicle's dimension on the motion planning for obstacle avoidance. For further tuning of the parameter used for motion planning, knowledge of the repulsive potential field is utilized to adapt the algorithm. In literature, the potential field is used to compute the target's attractive force and repulsive force for the obstacle. In the proposed algorithm, thresholds of the fuzzy membership function for the sensor group inputs are kept adaptive to the repulsive potential field. When the vehicle is away from the obstacle, the repulsive function is smaller, and the factors ' $\mathrm{K}$ ' are reduces so that the vehicle moves closer to the walls or the obstacle. If the vehicle has arrived at a safe distance from the obstacle, the repulsive function is kept as positive for the increment of factor ' $\mathrm{K}$ '. It keeps the vehicle away from the obstacle with the increase in thresholds of the fuzzy membership function.

A parking scenario with a static obstacle is developed in MATLAB software to demonstrate the proposed algorithm's practicality and efficacy. Different dimension of the CLMR is used to check the effect of the length and width on the static rule-based design for the motion planning controller. Also, the parking is shown as only forward parking, but the algorithm is generalized for reverse parking also. A MATLAB simulation environment of size 20 by $20 \mathrm{~ms}$ is created with a combination of a parking slot and a static obstacle in the path of the parking. Here, the static obstacle is assumed to appear during the runtime of the vehicle, and the vehicle does not have any prior information about the environment. It only has the start and finishes point of the parking path. The static obstacle is obstructing the road, and the vehicle has supposed to detour itself and navigate to the parking slot. $\mathrm{d}_{1 \mathrm{~s}}$ and $\mathrm{d}_{3 \mathrm{~s}}$ are set as 0.5 , and $\mathrm{d}_{2 \mathrm{~s}}$ are set as 1 .

Initially, the system shown in Figure 2 is tested for the two different vehicles with different dimensions without using an adaptive motion planning algorithm. For this, the constant membership functions for each variable [28] are used. This rule-base is a handcrafted rule-based design for obstacle avoidance during the parking maneuver. As the previous work is tested for the small hatchback vehicles, the membership functions are designed to work for all the passenger vehicles for them only. However, the system's results shown in Figure 5 show the difference in the motion planning for the larger vehicle with the same membership function used for the smaller vehicle. Figure 5(a) shows successful parking and detouring of the Hyundai i20 in the static obstacle's presence in the forward direction. But when the Range rover is tested for the same system, it collides with the obstacle, as shown in Figure 5(b). It shows that the inference of the near and medium distances has to be changed as per the vehicle's length and width. So, every time, it needs to build a different membership function for the different vehicle. Figure 5 illustrates the two paths followed by two different vehicles in the same system. Our proposed algorithm gives a generalized solution for the motion planning of any segment of the vehicle.

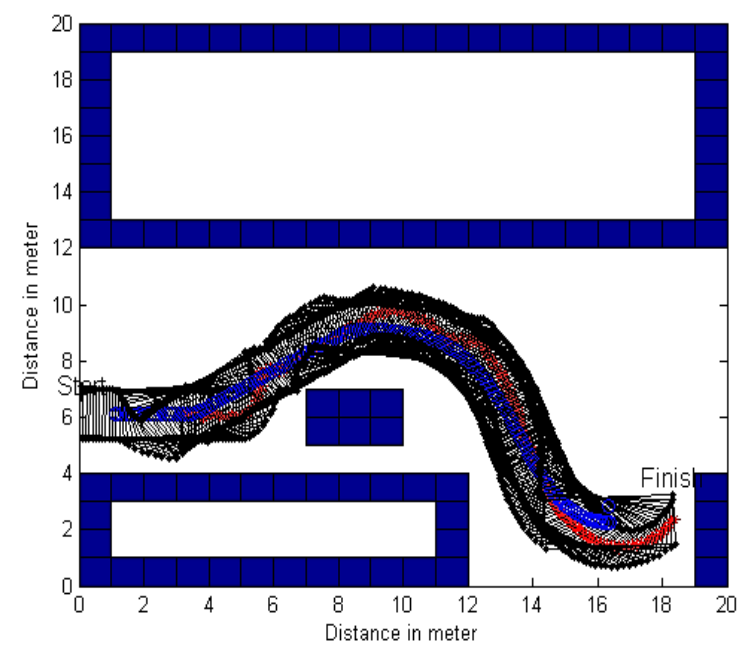

(a)

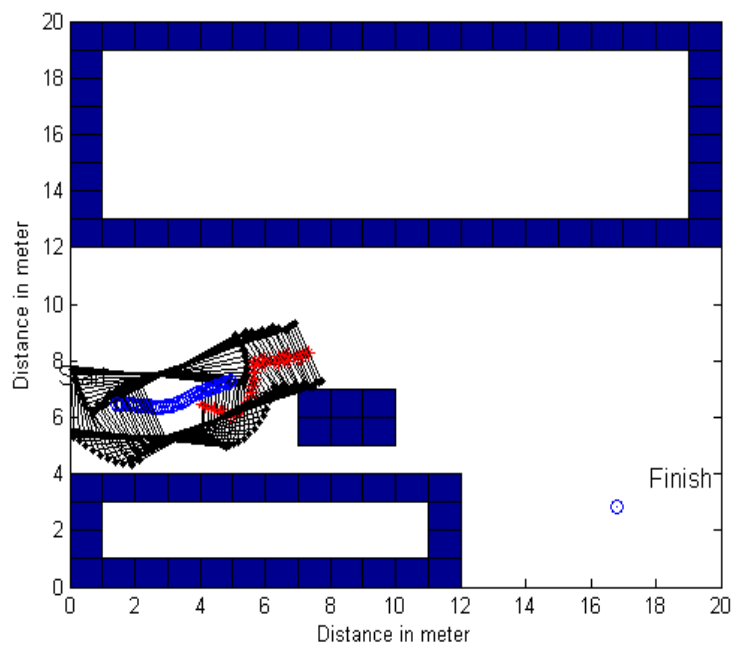

(b)

Figure 5. Motion planning of the CLMR in forward parking in the presence of the static obstacle with static membership function for; (a) Hyundai i20 (L-3996 mm and W-1734 mm) and (b) Range rover (L-5200 mm and $\mathrm{W}-2220 \mathrm{~mm}$ ) 
The forward parking for Hyundai i20 with the proposed adaptive motion planning algorithm is shown in Figure 6(a). It shows successful motion planning of the vehicle around the static obstacle. At each stepsequence, the adaptation in the three factors ' $\mathrm{K}_{1}$ ', ' $\mathrm{K}_{2}$ ', and ' $\mathrm{K}_{3}$ ' is shown in Figure $6(\mathrm{~b})$. It can be observed that initially, when the vehicle is safe away from the obstacle and the wall, both the $\mathrm{K}_{2}$ and $\mathrm{K}_{3}$ tend to decrease slightly so that vehicle moves closer to the wall. When it detects the front side obstacle, the algorithm increases the value of $\mathrm{K}_{2}$ for the adaptation of the front sensor membership function. An increment in $\mathrm{K}_{2}$ results in sharper detouring of the vehicle around the static obstacle. All three ' $\mathrm{K}$ ' parameters are adjusted to perform the motion planning corresponding to the vehicle's dimensions.

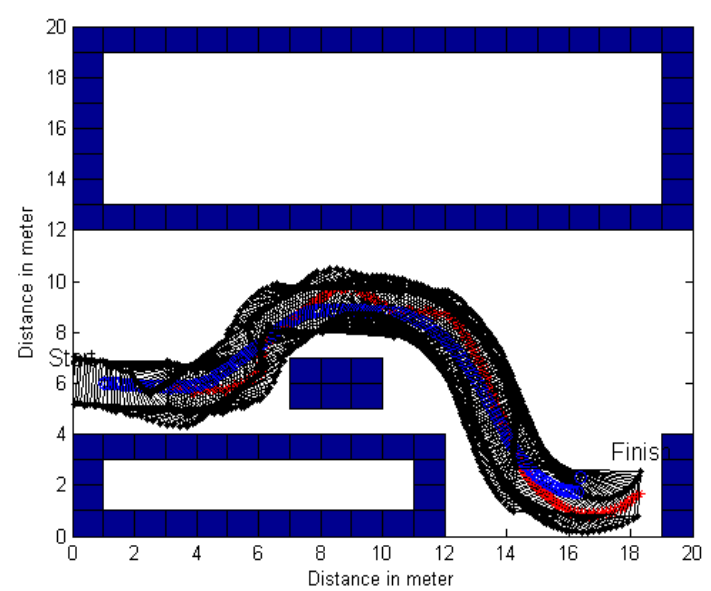

(a)

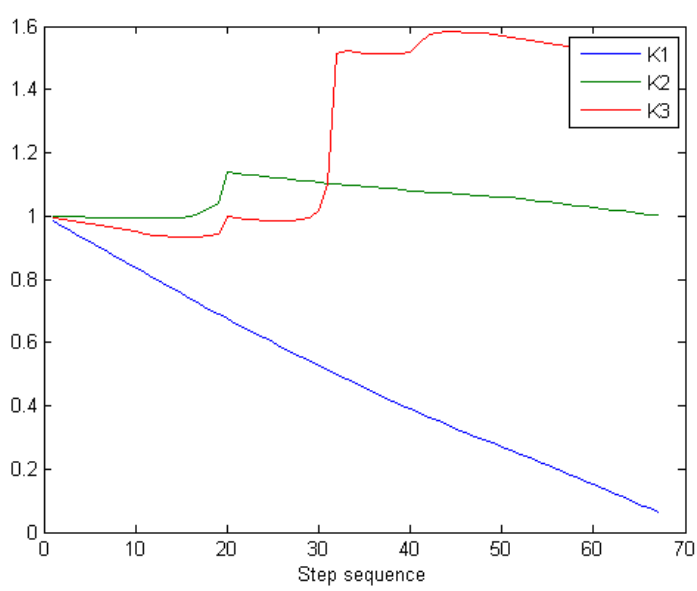

(b)

Figure 6. These figures are; (a) motion planning of the CLMR in forward parking in the presence of the static obstacle with the proposed algorithm for Hyundai i20 and (b) changes in the K1, K2, and K3

A similar result for the larger vehicle Range rover is shown in Figures 7(a) and 7(b). Figure 7(a) shows the larger vehicle's successful avoidance using the proposed algorithm compared to Figure 5(b). It can be noted that when the vehicle approaches the upper road boundary wall, the value of $\mathrm{K}_{1}$ increases that indicates the vehicle approaches near to the wall.

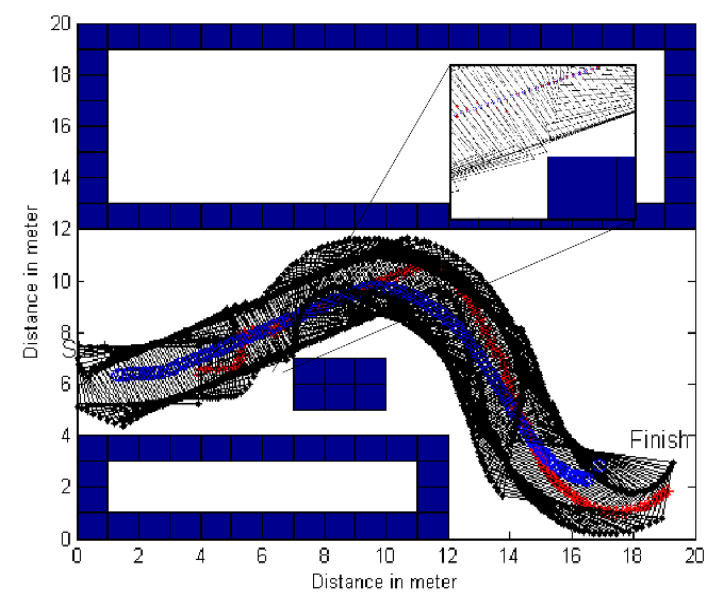

(a)

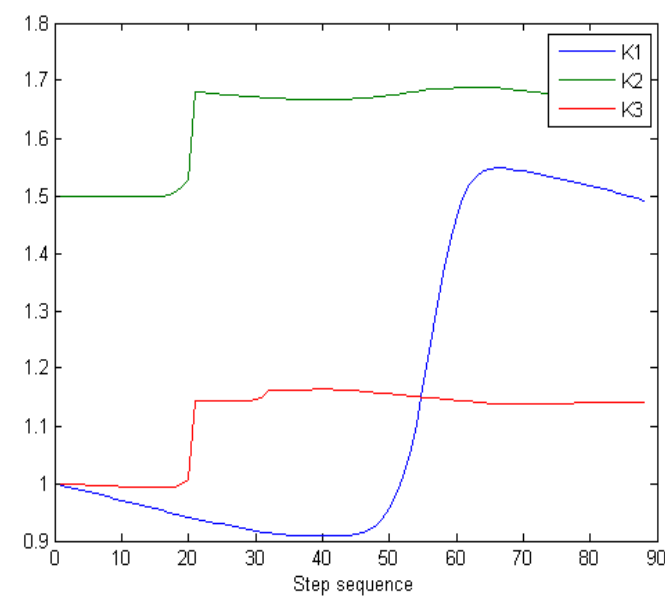

(b)

Figure 7. These figures are; (a) motion planning of the CLMR in forward parking in the presence of the static obstacle with the proposed algorithm for range rover and (b) changes in the $\mathrm{K}_{1}, \mathrm{~K}_{2}$, and $\mathrm{K}_{3}$

A previous work [25] shows the risk assessment for the obstacle avoidance using the potential field approach, as shown in Figure 8. Here the vehicle is supposed to move in valet parking, and it avoids another 
vehicle coming in front of the ego vehicle. This approach's major drawback is when the ego vehicle is taken with the larger size, and if there is a narrow passage between two vehicles, the risk assessment is higher. So, the vehicle may not follow a solution and stop due to excessive repulsive forces between the wall and the obstacle. However, our algorithm's adaptiveness makes it possible to go through the narrow passage by scaling down the inferences of the near, medium, and far membership function using K1, K2, and K3.

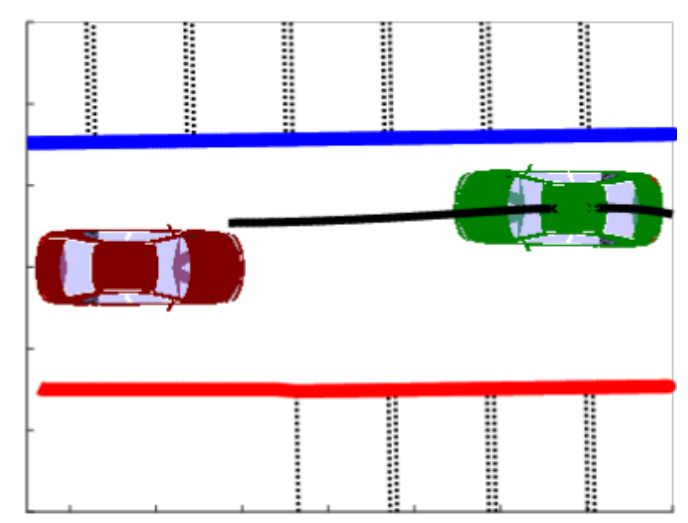

Figure 8. Motion planning using the repulsive potential field and RRT from [25]

A result of the proposed algorithm for the Range rover vehicle in the presence of the narrow passage is shown in Figure 9(a). The corresponding changes in $K_{1}, K_{2}$ and $K_{3}$ is also shown in Figure 9 (b). Using this approach, the limitation of the potential field is also addressed. Our algorithm utilized the knowledge of the potential field widely used in motion planning algorithms and adapted the fuzzy inference system for a better possible solution.

In summary, our proposed algorithm provides a unique solution for addressing the motion planning in autonomous parking systems to be used for the different segments of the vehicle. Due to the non-holonomic shape of the CLMR, path planning is challenging compared to Omni-directional robots. Our system tries to mimic the inference similar to the expert human driver up to a certain degree. In future work, further optimization of this adaptation by learning rate and initialization can be done.

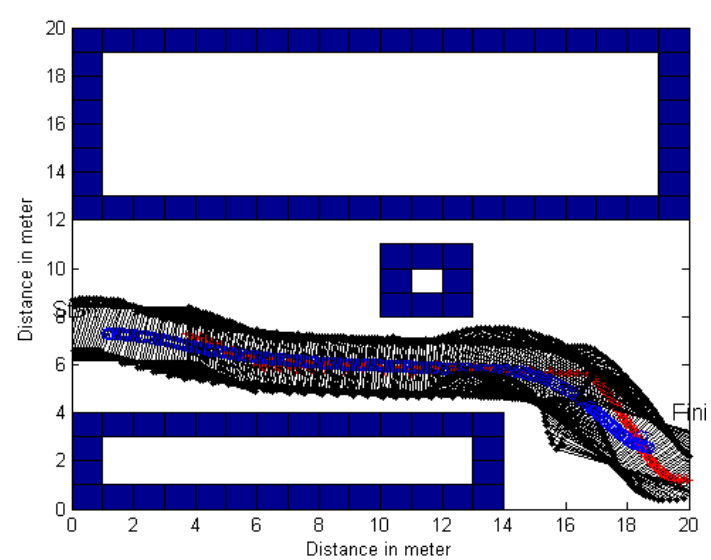

(a)

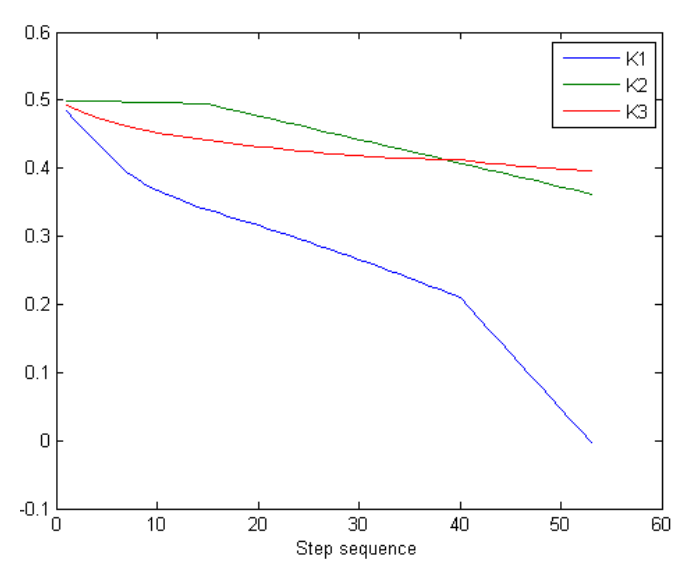

(b)

Figure 9. These figures are; (a) motion planning in the presence of narrow passage for the range rover and (b) corresponding changes in $\mathrm{K}_{1}, \mathrm{~K}_{2}$ and, $\mathrm{K}_{3}$

\section{CONCLUSION}

In this paper, a generalized solution of a motion planning-based parking system is derived that is adaptive enough to the vehicles' different dimensions. The system architecture integrates the navigation behavior into the standalone parking controller using ultrasonic sensors. The proposed algorithm intelligently adapts the fuzzy membership function utilizing the vehicle's actual dimension and the perceived sensor 
information from the localization of the vehicle. The simulation results indicate that the vehicle's motion path is adapted with the repulsive potential field's value. Also, three different factors make it possible to adjust the inputs of the system separately. When the narrow passing gap is available, lowering the values of K1, K2, and K3 can solve the problem. This adaptive algorithm that enables autonomous parking for any sized vehicle for scenarios including dynamic situations can be made available to solve real-time parking solutions.

\section{REFERENCES}

[1] S. J. Chang and T. H. S. Li, "Design and implementation of fuzzy parallel-parking control for a car-type mobile robot," Journal of Intelligent and Robotic Systems, vol. 34, no. 2, pp. 175-194, 2002, doi: 10.1023/A:1015664327686.

[2] Y. Zhao and E. G. Collins, "Robust automatic parallel parking in tight spaces via fuzzy logic," Robotics and Autonomous Systems, vol. 51, no. 2-3, pp. 111-127, 2005, doi: 10.1016/j.robot.2005.01.002.

[3] K. Demirli and M. Khoshnejad, "Autonomous parallel parking of a car-like mobile robot by a neuro-fuzzy sensorbased controller," Fuzzy Sets and Systems, vol. 160, no. 19, pp. 2876-2891, 2009, doi: 10.1016/j.fss.2009.01.019.

[4] B. Panomruttanarug and K. Higuchi, "Fuzzy Logic Based Autonomous Parallel Parking System with Kalman Filtering," SICE Journal of Control, Measurement, and System Integration, vol. 3, no. 4, pp. 266-271, 2010, doi: 10.9746/jcmsi.3.266.

[5] T. H. S. Li, Y. C. Yeh, J. D. Wu, M. Y. Hsiao, and C. Y. Chen, "Multi-functional intelligent autonomous parking controllers for car-like mobile robots," IEEE Transactions on Industrial Electronics, vol. 57, no. 5, pp. 1687-1700, 2010, doi: 10.1109/TIE.2009.2033093.

[6] S.-J. Huang and Y.-S. Hsu, "Parking Space Detection and Trajectory Tracking Control for Vehicle Auto-Parking," International Journal of Mechanical and Mechatronics Engineering, vol. 11, no. 10, pp. 1712-1718, 2017, doi: 10.5281/zenodo.1340376.

[7] J. Song, W. Zhang, X. Wu, H. Cao, Q. Gao, and S. Luo, "Laser-based SLAM automatic parallel parking path planning and tracking for passenger vehicle," IET Intelligent Transport Systems, vol. 13, no. 10, pp. 1557-1568, 2019, doi: 10.1049/iet-its.2019.0049.

[8] H. Ye, H. Jiang, S. Ma, B. Tang, and L. Wahab, "Linear model predictive control of automatic parking path tracking with soft constraints," International Journal of Advanced Robotic Systems, vol. 16, no. 3, pp. 1-13, 2019, doi: $10.1177 / 1729881419852201$.

[9] C. Li, H. Jiang, S. Ma, S. Jiang, and Y. Li, "Automatic parking path planning and tracking control research for intelligent vehicles," Applied Sciences, vol. 10, no. 24, pp. 1-27, 2020, doi: 10.3390/app10249100.

[10] W. Dongshu, Z. Yusheng, and S. Wenjie, "Behavior-based hierarchical fuzzy control for mobile robot navigation in dynamic environment," in 2011 Chinese Control and Decision Conference (CCDC), 2011, pp. 2419-2424, doi: 10.1109/CCDC.2011.5968614.

[11] O. Motlagh, S. H. Tang, N. Ismail, and A. R. Ramli, “An expert fuzzy cognitive map for reactive navigation of mobile robots,” Fuzzy Sets and Systems, vol. 201, pp. 105-121, 2012, doi: 10.1016/j.fss.2011.12.013.

[12] H. Mo, Q. Tang, and L. Meng, "Behavior-based fuzzy control for mobile robot navigation," Mathematical Problems in Engineering, vol. 2013, no. 1, 2013, doi: 10.1155/2013/561451.

[13] P. K. Mohanty and D. R. Parhi, "Navigation of autonomous mobile robot using adaptive network based fuzzy inference system," Journal of Mechanical Science and Technology, vol. 28, no. 7, pp. 2861-2868, 2014, doi: 10.1007/s12206-014-0640-2.

[14] A. Pandey, R. K. Sonkar, K. K. Pandey, and D. R. Parhi, "Path planning navigation of mobile robot with obstacles avoidance using fuzzy logic controller," in 2014 IEEE 8th International Conference on Intelligent Systems and Control (ISCO), 2014, pp. 36-41, doi: 10.1109/ISCO.2014.7103914.

[15] H. Omrane, M. S. Masmoudi, and M. Masmoudi, "Fuzzy Logic Based Control for Autonomous Mobile Robot Navigation," Computational Intelligence and Neuroscience, vol. 2016, no. 2, pp. 1-10, 2016, doi: $10.1155 / 2016 / 9548482$.

[16] F. Fathinezhad, V. Derhami, and M. Rezaeian, "Supervised fuzzy reinforcement learning for robot navigation," Applied Soft Computing, vol. 40, pp. 33-41, 2016, doi: 10.1016/j.asoc.2015.11.030.

[17] R. Zhao and H. K. Lee, "Fuzzy-based path planning for multiple mobile robots in unknown dynamic environment," Journal of Electrical Engineering and Technology, vol. 12, no. 2, pp. 918-925, 2017, doi: 10.5370/JEET.2017.12.2.918.

[18] M. Khatib, H. Jaouni, R. Chatila, and J. P. Laumond, "Dynamic path modification for car-like nonholonomic mobile robots," in Proceedings of International Conference on Robotics and Automation, vol. 4, pp. 2920-2925, 1997, doi: 10.1109/robot.1997.606730.

[19] W. L. Xu and S. K. Tso, "Sensor-based fuzzy reactive navigation of a mobile robot through local target switching," IEEE Transactions on Systems, Man, and Cybernetics, Part C (Applications and Reviews), vol. 29, no. 3, pp. 451-459, 1999, doi: 10.1109/5326.777079.

[20] M. M. Joshi and M. A. Zaveri, "Fuzzy based autonomous robot navigation system," in 2009 Annual IEEE India Conference, 2009, pp. 1-4, doi: 10.1109/INDCON.2009.5409419.

[21] H. Seki, Y. Kamiya, and M. Hikizu, "Real-Time Obstacle Avoidance Using Potential Field for a Nonholonomic Vehicle," Factory Automation, pp. 523-542, 2010, doi: 10.5772/9508.

[22] M. A. K. Jaradat, M. H. Garibeh, and E. A. Feilat, "Autonomous mobile robot dynamic motion planning using hybrid fuzzy potential field," Soft Computing, vol. 16, no. 1, pp. 153-164, 2012, doi: 10.1007/s00500-011-0742-z. 
[23] O. Montiel, U. Orozco-Rosas, and R. Sepúlveda, "Path planning for mobile robots using Bacterial Potential Field for avoiding static and dynamic obstacles," Expert Systems with Applications, vol. 42, no. 12, pp. 5177-5191, 2015, doi: 10.1016/j.eswa.2015.02.033.

[24] T. Y. Abdalla, A. A. Abed, and A. A. Ahmed, "Mobile robot navigation using PSO-optimized fuzzy artificial potential field with fuzzy control," Journal of Intelligent and Fuzzy Systems, vol. 32, no. 6, pp. 3893-3908, 2017, doi: 10.3233/IFS-162205.

[25] Y. Jeong, S. Kim, B. R. Jo, H. Shin, and K. Yi, "Sampling based vehicle motion planning for autonomous valet parking with moving obstacles," International Journal of Automotive Engineering, vol. 9, no. 4, pp. 215-222, 2018, doi: $10.20485 /$ jsaeijae.9.4_215.

[26] C. Y. Chou and C. F. Juang, "Navigation of an autonomous wheeled robot in unknown environments based on evolutionary fuzzy control," Inventions, vol. 3, no. 1, 2018, Art. no. 3, doi: 10.3390/inventions3010003.

[27] K. Shibata, N. Shibata, K. Nonaka, and K. Sekiguchi, "Model Predictive Obstacle Avoidance Control for Vehicles with Automatic Velocity Suppression using Artificial Potential Field," IFAC-PapersOnLine, vol. 51, no. 20, pp. 313-318, 2018, doi: 10.1016/j.ifacol.2018.11.050.

[28] N. Nakrani and M. Joshi, "Fuzzy based Autonomous Parallel Parking Challenges in Real time Scenario," in The International Symposium on Intelligent Systems Technologies and Applications-ISTA 2016, vol. 530, 2016, pp. 789-802, doi: 10.1007/978-3-319-47952-1_63.

\section{BIOGRAPHIES OF AUTHORS}

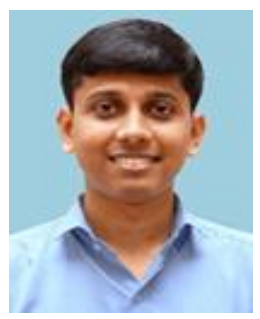

Naitik M. Nakrani is a Ph.D. Scholar in the field of Robotics and Machine learning. He has received B.E. in Electronics \& Communication from the Dharmsinh Desai University, Nadiad, in 2010, and received M.E. in Electronics \& Communication from Gujarat Technological University in 2012. He has worked as an Assistant professor in Electronics and Communication dept, UTU, for eight years. His research interests are autonomous systems, robotics, machine learning, and fuzzy system.

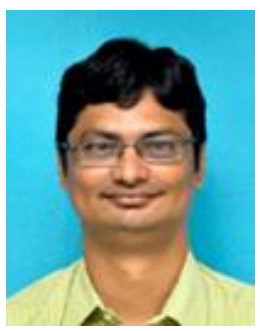

Dr. Maulin Joshi is currently serving as a professor and head at Sarvajanik College of Engineering and Technology, Surat. He completed his B.E. from DDIT Nadiad in 1996, Masters from IIT Bombay in 2005, and Ph.D. from SVNIT in 2012. He has 22 years of teaching experience at the U.G. and P.G. level and 17 years of research experience. He is a regular contributor to various peer-reviewed journals and conferences and serves on the various program committees and a reviewer for reputed journals. 